\title{
Black-hole radiation, the fundamental area unit, and the spectrum of particle species
}

\author{
Shahar Hod \\ The Racah Institute for Physics, The Hebrew University, Jerusalem 91904, Israel
}

(October 29, 2018)

\begin{abstract}
Bekenstein and Mukhanov have put forward the idea that, in a quantum theory of gravity a black hole should have a discrete mass spectrum with concomitant discrete line emission. We note that a direct consequence of this intriguing prediction is that compared with blackbody radiation, black-hole radiance is less entropic. We calculate the ratio of entropy emission rate from a quantum black hole to the rate of black-hole entropy decrease, a quantity which according to the generalized second law (GSL) of thermodynamics should be larger than unity. Implications of our results for the GSL, for the value of the fundamental area unit in quantum gravity, and for the spectrum of massless particles in nature are discussed.
\end{abstract}

One of the most remarkable theoretical predictions of the former century is Hawking's celebrated result that black holes are quantum mechanically unstable: they decay by the emission of a (filtered) thermal radiation [1]. According to Hawking's result, the black hole emits quanta of all frequencies, distributed according to the usual black-body spectrum (with a gray-body factor which represents the imprint of passage through the curvature potential surrounding the black hole).

However, Hawking's prediction of black-hole evaporation is at a semiclassical level in the sense that the matter fields are treated quantum mechanically, but the spacetime (and the black hole itself) are treated classically. One therefore suspects some modifications of the character of the radiation when quantum properties of the black hole itself are properly taken into account.

The quantization of black holes was proposed long ago by Bekenstein [2]. Based on the remarkable observation that the horizon area of a nonextremal black hole behaves as a classical adiabatic invariant, and in the spirit of Ehrenfest principle [3], any classical adiabatic invariant corresponds to a quantum entity with discrete spectrum, Bekenstein [2] conjectured that the horizon area of a quantum black hole should have a discrete eigenvalue spectrum of the form

$$
A_{n}=\gamma \ell_{P}^{2} \cdot n \quad ; \quad n=1,2, \ldots,
$$

where $\gamma$ is a dimensionless constant, and $\ell_{P}=\left(\frac{G}{c^{3}}\right)^{1 / 2} \hbar^{1 / 2}$ is the Planck length (we use gravitational units in which $G=c=1$ ). This type of quantization-law has since been revived on various grounds [4] 23] (most of these derivations have been made in the last few years). In particular, Mukhanov and Bekenstein [4 6] used a combination of thermodynamic (the area-entropy relation $S_{B H}=A / 4 \hbar$ for black holes) and statistical physics (the BoltzmannEinstein formula) arguments, and found that the dimensionless constant $\gamma$ in Eq. (11) should be of the form $\gamma=4 \ln \beta$, with $\beta=2,3, \ldots$ (this corresponds to a degeneracy factor of $\beta^{n}$ for the $n$th area level). Using Bohr's correspondence principle Hod [16] has recently given evidence in favor of the value $\beta=3$.

The discrete mass (area) spectrum implies a discrete line emission from a quantum black hole; the radiation emitted by the black hole will be at integer multiples of the fundamental frequency $\omega_{0}=\ln \beta / 8 \pi M[5]$.

We note that a direct consequence of the discrete spectrum is that compared with blackbody radiation, black-hole radiance is less entropic. In fact, one expects the entropy of the radiation emitted by the black hole (or equivalently, the total number of configurations evaporated by the black hole) to decrease as $\beta$ increases: The entropy of the radiation should be maximal when the various transitions have equal probabilities, but the fundamental transition $n \rightarrow n-1$ becomes more and more dominant as the value of $\beta$ increases.

The entropy of a system measures one's lack of information about its actual internal configuration [24]26]. Suppose that all that is known about the system's internal configuration is that it may be found in any of a number of states, with probability $p_{n}$ for the $n$th state. Then the entropy associated with the system is given by Shannon's well-known relation $S=-\sum_{n} p_{n} \ln p_{n}$.

The probability for a black hole to emit a specific quantum should be proportional to the degeneracy of the final black-hole quantum state, to the gray-body factor $\Gamma$ (representing a scattering of the quantum off the spacetime curvature surrounding the black hole), and to the square of the matrix element. In the spirit of the original treatment of Bekenstein and Mukhanov [5] we assume that the matrix element does not vary much as one goes from a nearest neighbor transition to one between somewhat farther neighbors. Thus, aside from an overall normalization factor, the matrix element does not enter into our simple estimate. This assumption is further supported by a recent analysis of Massar and Parentani [27]. Thus, the probability $p_{k}$ to jump $k$ steps in the mass (area) ladder is proportional to $\Gamma(k) \beta^{-k}$. 
A toy model. To gain some insight into the physical problem, we shall first consider a simplified toy model. It is well known that for massless fields, $\Gamma(M \omega)$ approaches 0 in the low-frequency limit, and has a high-frequency limit of 1 [28]. A rough approximation of this effect can be achieved by introducing a low frequency cutoff at some $\omega=\omega_{c}$ [29]. That is, $\Gamma(\varpi)=0$ for $\varpi<\varpi_{c}$ and $\Gamma(\varpi)=1$ otherwise, where $\varpi \equiv M \omega$.

The ratio $R=\left|\dot{S}_{\text {rad }} / \dot{S}_{B H}\right|$ of entropy emission rate from the quantum black hole to the rate of black-hole entropy decrease is given by

$$
R=\frac{-\sum_{i=1}^{N_{s}} \sum_{k=1}^{\infty} C \Gamma(k) \beta^{-k} \ln \left[C \Gamma(k) \beta^{-k}\right]}{\sum_{i=1}^{N_{s}} \sum_{k=1}^{\infty} C \Gamma(k) \beta^{-k} k \ln \beta},
$$

where $N_{s}$ is the effective number of (massless) particle species emitted ( $N_{s}$ takes into account the various modes emitted), and $C$ is a normalization factor, defined by the normalization condition

$$
\sum_{i=1}^{N_{s}} \sum_{k=1}^{\infty} C \Gamma(k) \beta^{-k}=1 .
$$

Equation (2) for $R$ yields

$$
R=1-\frac{\ln \left[\left(1-\beta^{-1}\right) \beta^{k_{0}} / N_{s}\right]}{\ln \beta} \frac{\beta-1}{1+k_{0}(\beta-1)},
$$

where $k_{0} \equiv \omega_{c} / \omega_{0}$. As expected, the number of configurations evaporated by the black hole (or equivalently, the value of $R$ ) increases with increasing number $N_{s}$ of massless particles in nature.

For the emission process to respect the GSL, $R$ should be larger than (or equal) unity. Thus, one finds that the number $N_{s}$ of massless particles should satisfy the relation

$$
\left(1-\beta^{-1}\right) \exp \left(8 \pi \varpi_{c}\right) \leq N_{s} .
$$

In accordance with our previous expectation, the entropy of the radiation emitted by the black hole decreases as the value of $\beta$ increases. Thus, the minimal value allowed for $N_{s}$ increases with increasing value of $\beta$.

One can estimate the maximally allowed value of $\beta$ (which is consistent with the GSL) by taking $\varpi_{c} \simeq 0.2$ (the location of the peak in the total power spectrum [28]), and $N_{s} \simeq 112$ (this estimate takes into account the modes which make the dominant contribution to the black-hole spectrum [28]: three species of neutrinos with modes having $j=1 / 2,3 / 2,5 / 2$, a photon with modes having $j=1,2$, and a graviton with modes having $j=2,3)$. Taking cognizance of Eq. (5) one finds $\beta_{\max } \simeq 3.7$ (which in practice implies a maximal value of 3 for $\beta$ ). An exact calculation to be given below reveals that this is a fairly good estimate.

An exact calculation. The most significant correction to our toy-model will result from the mode-dependent of the gray-body factor $\Gamma_{s, \varpi, j, m}$ (first calculated numerically by Page [28]), which is a function of the particle species $s$, the frequency $\varpi$, and the angular-momentum $(j, m)$.

We consider the emission of a canonical set of three species of neutrinos, photon, and a graviton. The ratio $R=\left|\dot{S}_{\text {rad }} / \dot{S}_{B H}\right|$ of entropy emission rate from the quantum black hole to the rate of black-hole entropy decrease is given in Table [. For the evaporation process to respect the GSL, $R$ should be larger (or equal) than unity. We therefore conclude that the maximally allowed value for $\beta$ is 3 (see Table $\mathbb{1}$ ).

Summary and physical implications. We have studied the emission of radiation from a quantized black hole, which is characterized by a discrete mass spectrum. [The discrete mass spectrum (with an evenly spaced area levels) has been

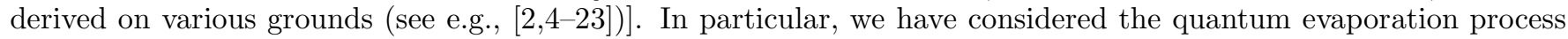
from the point of view of the generalized second law of thermodynamics. It was conjectured, and demonstrated explicitly by a simplified toy-model, that the entropy emitted from the black hole decreases as the area spacing increases. This allowed us to derive an upper bound to the value of the fundamental area spacing $\Delta A=4 \ell_{P}^{2} \ln 3$. Remarkably, this value agrees with the one recently derived based on Bohr's correspondence principle [16].

We note, however, that in recent years there is a growing evidence that neutrinos have finite masses. This implies that black holes with masses larger than $\hbar / m_{\nu}$ will not emit neutrinos with mass $m_{\nu}$ in a significant rate. As a direct consequence, the entropy emitted from the black hole would decrease because the aveliable phase space becomes smaller. In fact, we learn form Table 1 that if there were only two kinds of massless neutrinos species in nature, then the largest value allowed for $\beta$ (which is still consistent with the GSL) is 2. (The GSL would be violated if there was no more than one massless neutrino specie.)

In view of the previous discussion, we see only few possible solutions to the apparent violation of the GSL: 
(1) The uniformally spaced area spectrum, first suggested by Bekenstein [2] (and rederived on various different grounds by many authors (4 23), does not hold in reality.

(2) The quantum matrix elements vary considerably as one goes from a nearest neighbor transition to one between farther neighbors. (We recall that we assumed in the spirit of the original analysis of Bekenstein and Mukhanov [5], and in accordance with the recent analysis of Massar and Parentani [27], that the quantum matrix element does not vary much as one goes from a nearest neighbor transition to one between farther neighbors.)

(3) Neutrinos are massless after all. In this case we have shown that the GSL is respected during the evaporation process (provided $\beta \leq 3$ ).

(4) There exists a yet-unknown massless particle(s) in nature apart from the photon and the graviton. This would increase the number of configurations (or equivalently, the entropy) evaporated by the black hole.

Evidently, this solution to the apparent violation of the GSL is much more 'exotic' than the former ones. However, one should not rule it out; In fact W. Pauli [30] proposed the existence of the neutrino itself as a "desperate" solution in order to prevent a violation of the law of the conservation of energy during the process of beta-decay. Here we conjecture the existence of a yet-unknown massless particle(s) in order to prevent a violation of another sacred law of physics, the (generalized) second law. The conjectured particle may therefore be appropriately named the 'entropon'.

\section{ACKNOWLEDGMENTS}

I thank Jacob D. Bekenstein for helpful discussions. This research was supported by a grant from the Israel Science Foundation.

[1] S. W. Hawking, Commun. Math. Phys. 43, 199 (1975).

[2] J. D. Bekenstein, Lett. Nuovo Cimento 11, 467 (1974).

[3] See for example M. Born, Atomic Physics (Blackie, London, 1969), eighth edition.

[4] V. Mukhanov, JETP Lett. 44, 63 (1986).

[5] J. D. Bekenstein and V. F. Mukhanov, Phys. Lett. B 360, 7 (1995).

[6] J. D. Bekenstein in XVII Brazilian National Meeting on Particles and Fields, eds. A. J. da Silva et. al. (Brazilian Physical Society, Sao Paulo, 1996), J. D. Bekenstein in Proceedings of the VIII Marcel Grossmann Meeting on General Relativity, eds. T. Piran and R. Ruffini (World Scientific, Singapore, 1998).

[7] P. O. Mazur, Phys. Rev. Lett. 57, 929 (1987).

[8] Ya. I. Kogan, JETP Lett. 44, 267 (1986).

[9] M. Maggiore, Nucl. Phys. B 429, 205 (1994).

[10] C. O. Lousto, Phys. Rev. D 51, 1733 (1995).

[11] Y. Peleg, Phys. Lett. B 356, 462 (1995).

[12] J. Louko and J. Mäkelä, Phys. Rev. D 54, 4982 (1996).

[13] A. Barvinsky and G. Kunstatter, Phys. Lett. B 389, 231 (1996).

[14] H. A. Kastrup, Phys. Lett. B 385, 75 (1996).

[15] J. Mäkelä, Phys. Lett. B 390, 115 (1997).

[16] S. Hod, Phys. Rev. Lett. 81, 4293 (1998).

[17] S. Hod, Phys. Rev. D 59, 024014 (1999).

[18] S. Hod, Gen. Rel. Grav. 31, 1639 (1999).

[19] C. Vaz and L. Witten, Phys.Rev. D 60, 024009 (1999).

[20] H. A. Kastrup, e-print gr-qc/9906104.

[21] M. Bojowald and H. A. Kastrup, e-print hep-th/9907043.

[22] D. V. Ahluwalia, Int. J. Mod. Phys. D 8, 651 (1999).

[23] R. Garattini, e-print gr-qc/9910037; e-print gr-qc/000309.

[24] C. E. Shannon and W. Weaver, The Mathematical Theory of Communications (University of Illinois Press, Urbana, 1949).

[25] E. T. Jaynes, Phys. Rev. 106, 620 (1957); 108, 171 (1957).

[26] J. D. Bekenstein, Phys. Rev. D 7, 2333 (1973).

[27] S. Massar and R. Parentani, e-print gr-qc/9903027, Nucl. Phys. B (to be published).

[28] D. N. Page, Phys. Rev. D 13, 198 (1976); Phys. Rev. D 14, 3260 (1976); Phys. Rev. D 16, 2402 (1977).

[29] W. H. Zurek, Phys. Rev. Lett. 49, 1683 (1982).

[30] W. Pauli, Phys. Rev. 38, 579 (1931). 
TABLE I. The ratio $R=\left|\dot{S}_{\text {rad }} / \dot{S}_{B H}\right|$ of entropy emission rate from a quantum black hole to the rate of black-hole entropy decrease.

\begin{tabular}{lll}
\hline \hline$\beta$ & $N \nu=2$ & $N \nu=3$ \\
\hline 2 & 1.042 & 1.119 \\
3 & 0.940 & 1.016 \\
4 & 0.888 & 0.962 \\
\hline \hline
\end{tabular}

